\title{
Fault Location and Compensation of the Harmonic Content of the Residual Fault Current during Single-Phase to Ground Faults in Compensated Networks
}

\author{
András M. Dán ${ }^{1}$ Dávid Raisz ${ }^{1}$ \\ ${ }^{1}$ Department of Electric Power Engineering (Power Systems and Environment Group) \\ Budapest University of Technology and Economics \\ Egry J. u. 18. H-1111, Budapest, Hungary \\ Fax: +3614633600 \\ dan.andras@vet.bme.hu, raisz.david@vet.bme.hu
}

\begin{abstract}
Ground fault location in compensated networks has been considered as a challenging task until recently. Several methods have been elaborated, most of them have not been able to prove their capability under field conditions, or they are too expensive or complicated.

This paper presents a new fault location technique, which uses current injection parallel to the Petersen coil. Simulation and field test results show the accuracy of the method. The injecting device is further used to decrease the harmonics in the residual fault current. This zero sequence active filtering method is analyzed in detail using computer simulations and compared to field test results.
\end{abstract}

\section{Key words}

Fault location, Active filters, Harmonic distortion, Power distribution reliability, Power quality

\section{Introduction}

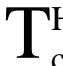
HE Hungarian rural medium voltage network is a compensated overhead line system with a total length of about $40000 \mathrm{~km}$. Fault statistics of this system pointed out that more than $90 \%$ of the total faults originates from single-phase to ground faults. About $90 \%$ of these faults are through an arc which gives the chance to achieve a self extinction due to the compensation by the Petersen coil (resonance grounding). The compensation is an automatic process, and is operating in some percent overcompensation.

Two important aspects of such networks are discussed in the paper.

\section{Fault location}

Since the residual fault current is below 10-15 A, a resistance has to be switched in parallel to the Petersen coil for cca. 2 seconds. This ensures that the fault current will be greater than the trigger level of the overcurrent protection, and thus the faulted line can be found and switched off. Once the faulted line is found, and assuming that the fault was not extinguished by the compensation, it is a time-consuming task to find the approximate location of the fault, and - in addition - it comprises several switching operations that can have a severe impact on voltage quality at consumers supplied from non-faulted lines.

\section{Maintained operation during the fault}

The continuous operation of the system can be maintained during a single-phase to ground fault if some requirements are fulfilled. The residual current at the fault location has to be kept securely below $12 \mathrm{~A}$ in order to ensure, that the touch voltage should remain below $65 \mathrm{~V}$ during the steady state faulty operation. (This assumption considers a $5 \Omega$ grounding resistance of the pole.)

Measurements are carried out regularly at the substations in order to check the above condition. In recent years these measurements showed, that in spite of having set the compensation to some 1-2 A fundamental residual current, the RMS of the residual current is in some substations greater than the allowed value. The analysis of the Fourier spectrum of the residual current shows that in these cases the harmonic content of the residual current is several times greater than the fundamental component. The circumstances resulting in the increasing of the harmonic content of the arc current and the possible solutions of the problem were outlined in [1] and [2].

In [3] the authors discussed the possible solutions for compensating the harmonic content of the residual current based on computer simulations.

\section{Fault location methods: state of the art}

Several solutions have been elaborated for determining fault locations and fault resistance. The most widespread alternatives are so-called fundamental frequency $(50 \mathrm{~Hz}$ or $60 \mathrm{~Hz}$ ) methods, however, all such methods are deficient in that at least two different operating states are required, which cannot be concurrently established and, consequently, that changes in certain quantities at fundamental frequency between the two measurements impact on the measured quantities thus making computation overly inaccurate for example:

- in the case of power lines running on the same set of poles, the load change occurring on the non-faulted line 
induces a voltage in the faulted one thus falsifying the measurement being done thereon,

- during a short-circuit existing on the medium voltage network (while measurements are being done), any shortcircuit happening on the high-voltage side results in a zero sequence induction in medium voltage thus falsifying the measurement being done on the medium voltage side,

- the load varies on the faulted or non-faulted branches.

An additional deficiency of calculation involving fundamental frequency quantities is that, for the benefit of accuracy, measurement needs to be performed for a long time (several seconds) due to the creation of various operating states so, during measurement, there is a considerable probability of

- the above-mentioned phenomena occurring,

- the fault location's impedance value changes, which likewise usually degrades accuracy in fault location determination.

A deterministic direct method is described in [5], however, this can only be used in the case of dedicated cable lines. It relies on computer simulation and assumes knowledge of all parameters as well as homogeneity over the entire measured length. This known solution is deficient in that it considers only fundamental frequency (e.g. $60 \mathrm{~Hz}$ ) signals, furthermore, it does not take into account any measurement uncertainties, under realistic circumstances probably works at a high error rate due to computer simulation being used, and is suitable only for determining cable faults so it does not facilitate use on overhead lines or mixed networks.

A procedure described in [6] likewise represents the state of the art and allows calculation of the fault location distance in the case of single-phase to ground faults. The procedure employs a fundamental frequency signal. Measurement is based on forced changing of the zero sequence impedance, utilizing measurement results obtained for the two different values thereof. Consequently, the fault has to exist for a relatively long duration and, due to the use of a lumped parameter model, experience shows that its accuracy is lower than if distributed parameter lines were used. Additionally, the procedure does not take into account positive and negative sequence capacities whose ignorance likewise reduces accuracy.

The procedure described in [7] involves detection of single-phase to ground faults on radial distribution lines. The solution has the same disadvantage as the method described in [6].

[8] likewise deals with fault location which essence is that a loop is created for a short duration using the defective and a non-faulted branch whenever there is a fault and that the fault location is determined based on information (numerical measurement data) that can be obtained from the loop configuration. The deficiency of this known solution is that conditions for creating a loop are not given in every case, as mentioned in [7], furthermore, due to the usage of fundamental frequency (e.g. $50 \mathrm{~Hz}$ ) quantities, the disadvantages already summarized in the introduction also appear in this solution.

A procedure described in [9] is used to determine the location of single-phase to ground faults in electric distribution networks by processing transient signals. This solution is deficient in that the transient process depends on the fault location's resistance, which renders this procedure inaccurate, therefore, its use is not expedient, which is even referred to in several later patent descriptions.

The procedure and equipment described in [10] is used to locate ground faults and to determine the distance thereof. This method involves estimation of the fault location resistance based on fundamental frequency zero sequence voltage and current and fundamental frequency phase voltage, therefore, the result is expected to be inaccurate. In addition, the disadvantages described in the introduction also appear in this method.

Fault location on electric lines described in [11] is based on the travelling wave reflection method. The deficiency of this known solution is that measurement needs to be first conducted on a non-faulted, switched off and nonoperating line, for example during commissioning, which makes subsequent installation circumstantial and the measurements need to be repeated for example whenever a new side-line is installed, furthermore, a generator suitable for creating and coupling impulses shorter than $2 \mathrm{~ns}$ is required, which likewise makes practical use difficult and significantly raises costs.

\section{Fault Location Using Current injection}

In the present paper a new method is proposed, where fault location is performed by injecting current in parallel to the Petersen coil. The frequency of the injected current is chosen so that it does not coincide with any of the harmonic or ripple control frequencies that are present at the network. (The injected current can be composed of multiple frequencies.) Therefore this method is independent from the disturbing effects of external circumstances to a great degree.

For the benefit of continuous power supply, this method is implemented economically, employing a simple design, adapted to the substation protection automation system; it is more accurate than the known solutions, employing real-time measurements of relatively short duration. (The measurement takes approx. $1.5 \mathrm{~s}$, which is smaller than the time allowed before the resistance is switched parallel to the Petersen coil.) Zero sequence voltages and currents measured in the substation are the input signals to the calculation procedure. The method is described in detail in [12].

\section{Simulation and Sensitivity Analysis}

A $22 \mathrm{kV}$ overhead line network has been modeled, which was fed by a transformer having a nominal power of $S_{n}=40$ MVA and a drop of $\varepsilon=10 \%$; the R/X was taken 0.1 .

The geometry of the overhead line can be seen in Fig. 1: 

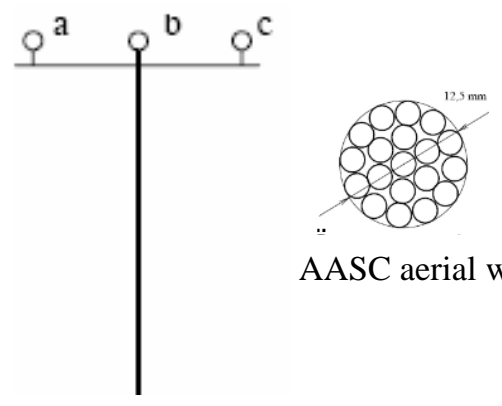

AASC aerial wire

Fig. 1. Overhead line geometry used for simulations

Pole height: $9 \mathrm{~m}, \mathrm{Dab}=\mathrm{Dbc}=1.3 \mathrm{~m}$, the nominal crosssection of the line was $95 \mathrm{~mm}^{2}(\mathrm{Al})$, the radius $\mathrm{r}=$ $0.65 \mathrm{~cm}(\mathrm{GMR}=0.757 * \mathrm{r}), \mathrm{R}_{\mathrm{DC}}=0.36 \mathrm{Ohm} / \mathrm{km}$.

The ground resistivity was $20 \Omega \mathrm{m}$, and the skin effect was taken into consideration.

TABLE I. shows the calculated line parameters.

TABLE I. Specific parameters of the line

\begin{tabular}{|c|c|c|c|c|}
\hline & $\begin{array}{l}\text { Freq. } \\
\mathrm{Hz}\end{array}$ & $\begin{array}{l}\text { Positive } \\
\text { sequence }\end{array}$ & $\begin{array}{c}\text { Zero } \\
\text { sequence }\end{array}$ & \\
\hline \multirow{3}{*}{$\mathrm{R}$} & 50 & 0.3602 & 0.5021 & $\Omega / \mathrm{km}$ \\
\hline & 400 & 0.3698 & 1.4293 & $\Omega / \mathrm{km}$ \\
\hline & 500 & 0.3751 & 1.6837 & $\Omega / \mathrm{km}$ \\
\hline \multirow{3}{*}{$\mathrm{L}$} & 50 & \multirow{3}{*}{1.1616} & 4.5059 & $\mathrm{mH} / \mathrm{km}$ \\
\hline & 400 & & 3.9203 & $\mathrm{mH} / \mathrm{km}$ \\
\hline & 500 & & 3.8604 & $\mathrm{mH} / \mathrm{km}$ \\
\hline $\mathrm{C}$ & $\begin{array}{c}50,400 \\
500\end{array}$ & 10.150 & 4.4574 & $\mathrm{nF} / \mathrm{km}$ \\
\hline
\end{tabular}

The substation was supposed to be feeding 20 lines of $50 \mathrm{~km}$ length each. The non-faulted lines were modeled as capacitances. The Petersen coil had a rating of $53 \mathrm{~A}$ (237 Ohm, $755 \mathrm{mH}$ ).

Fault locations were simulated at 2, 10, 20 and $48 \mathrm{~km}$ of one chosen line.

Simulations have shown, that using the proposed current injection method the accuracy of fault location is extremely good, below $200 \mathrm{~m}$.

Then a sensitivity analysis has been performed. Several sources of error have been investigated, two of which will be shown in the sequel.

\section{A) Errors resulting from inaccurate line parameters}

It was assumed that the parameters of the overhead lines are those shown in TABLEI, but the parameter calculation yields inaccurate results. All parameters have been varied randomly around the real value using a normal distribution shown in Fig. 2. The parameter variations have been performed independently of each other, and all the parameters have been perturbed at the same time. Several hundred cases have been investigated; Fig. 3 shows the distribution of the fault location calculation error in per cent. In $75 \%$ of the simulated cases the fault location accuracy was below $5.8 \%$.

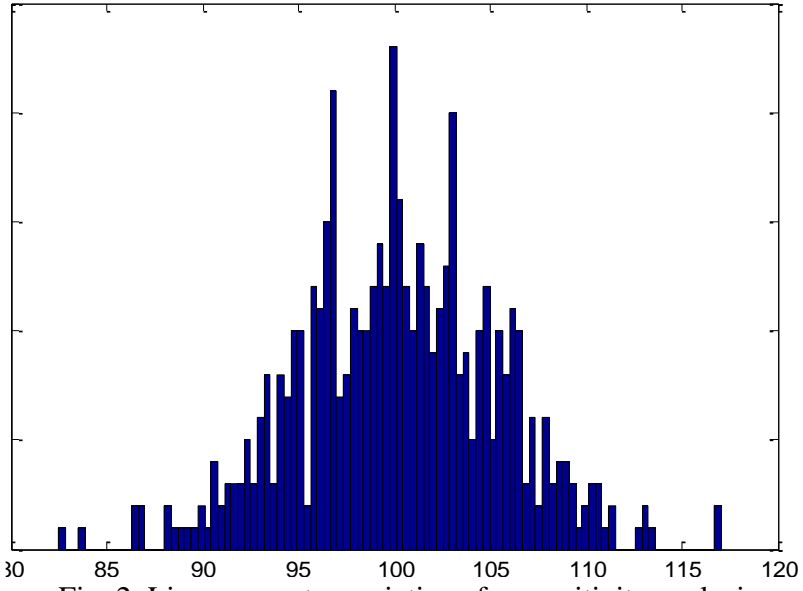

Fig. 2. Line parameter variations for sensitivity analysis (Relative frequency vs. parameter value in $\%$ of the real value)

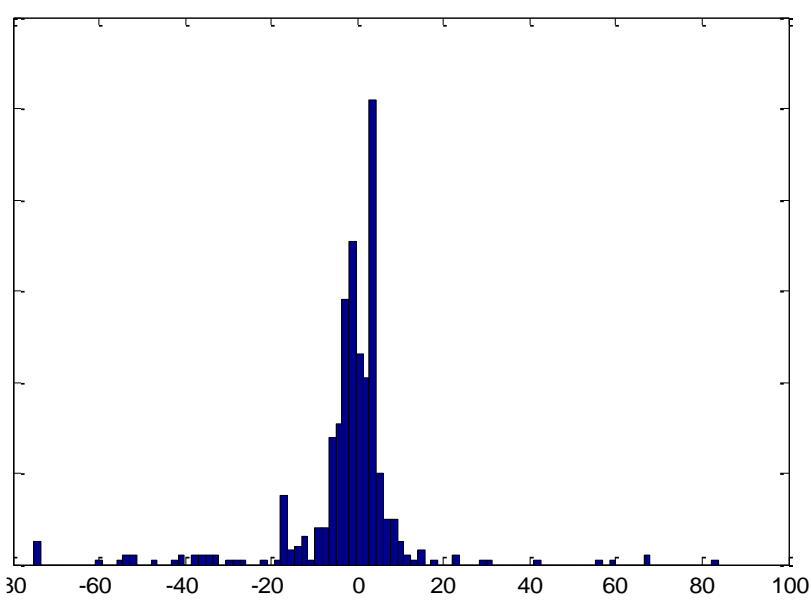

Fig. 3. Result of fault location accuracy simulations (Relative frequency vs. per cent deviation of the calculated fault location from the simulated one)

B) Errors resulting from inaccurate measurement of voltage and current phase angles

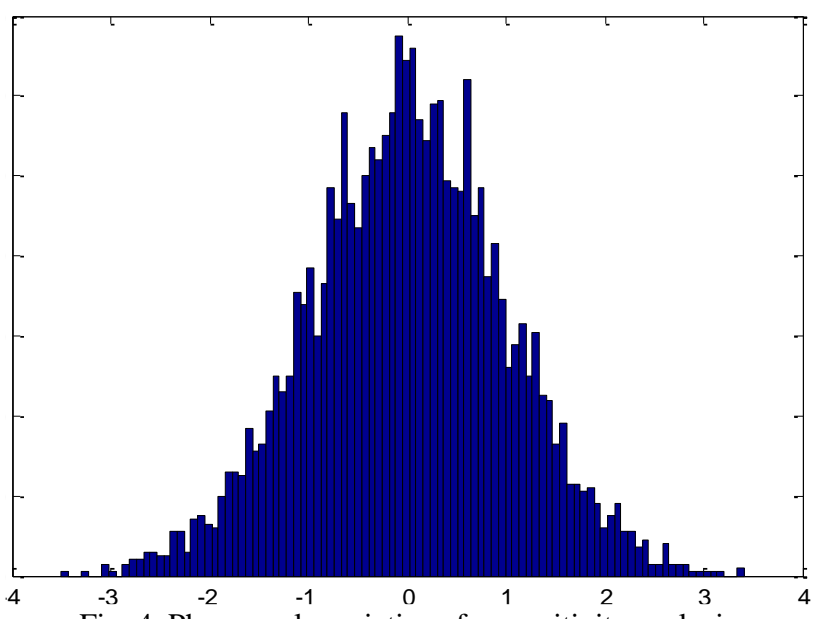

Fig. 4. Phase angle variations for sensitivity analysis (Relative frequency vs. deviation from the correct value in degrees)

In this case it was assumed that the correct line parameter values are known, but the phase angle measurements are inaccurate. All measured voltage and current phase angles have been varied independently, concurrently and randomly around their correct value using a normal distribution shown in Fig. 4. 
Several hundred cases have been investigated; Fig. 5 shows the distribution of the fault location calculation error in per cent. In $75 \%$ of the simulated cases the fault location accuracy was below $4.2 \%$.

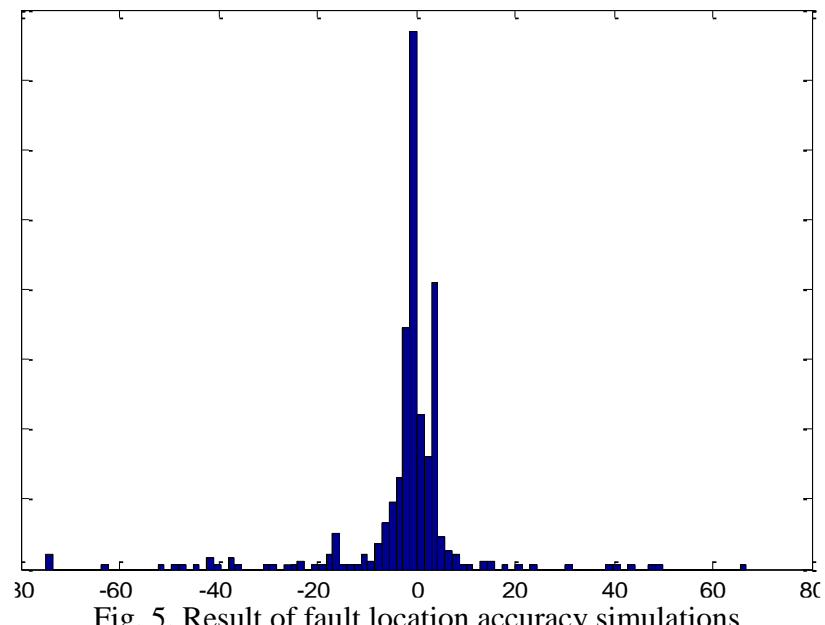

Fig. 5. Result of fault location accuracy simulations

(Relative frequency vs. per cent deviation of the calculated fault location from the simulated one)

\section{Field test results}

Several field tests have been carried out using artificial earth faults several kilometers away from the substation. Fig. 6 shows a section of simplified $22 \mathrm{kV}$ line scheme with a feeder (vertical line at the bottom) and several side-lines. The $132 / 22 \mathrm{kV}$ transformer (not shown in the Figure) is supplying the line from the bottom left of the feeder.

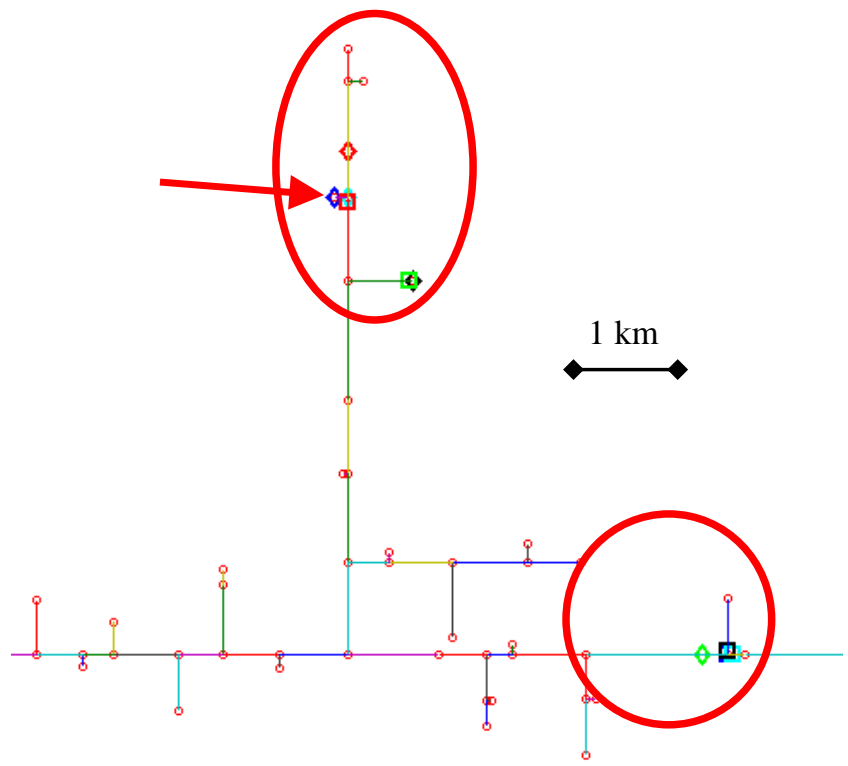

Fig. 6. Result of a field test measurement: possible fault locations are shown in a simplified network scheme, prioritized by their probability (the artificial fault was set up where marked by an arrow)

The field tests have yielded promising results. Though the method provides several possible fault locations (e.g. because it cannot distinguish between electrically identical side-lines based on substation measurements), these are prioritized, and the best results have been found to be very close to the artificial fault location see Fig. 6.)
Based on the encouraging field test results two prototypes have been installed at distinct substations, and are being tested for accuracy under real-life conditions.

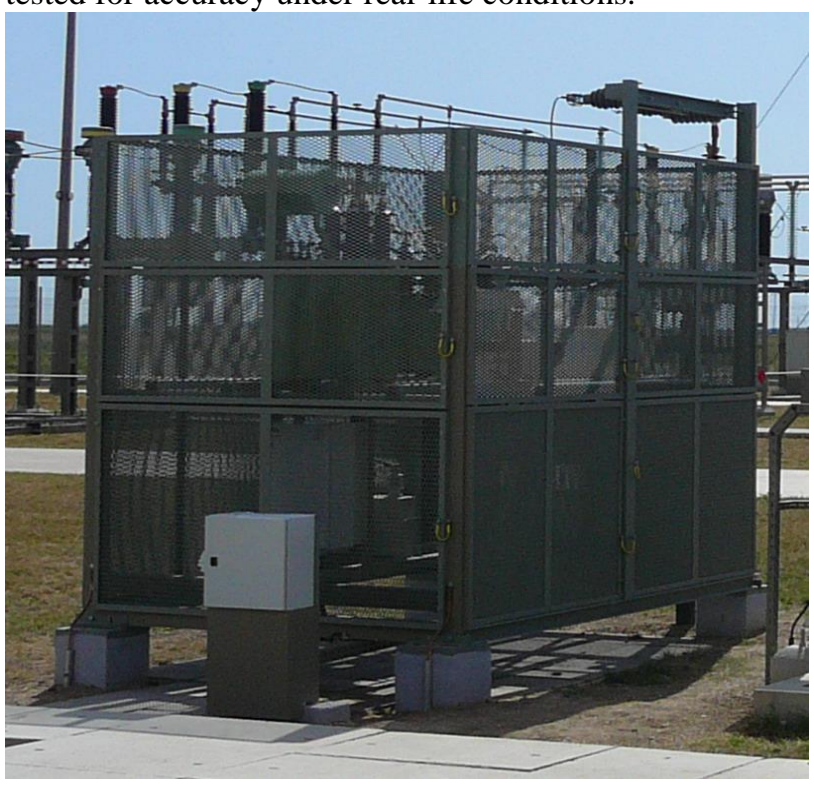

Fig. 7. Primary equipment at installation site No.1.

\section{Maintained Operation during Earth Faults}

Zero sequence active filtering has been proven to be an appropriate method for decreasing the harmonic content of the fault current near to zero [4]. By means of a zero sequence harmonic current generator the control system forces the zero sequence harmonic voltages to be the opposite of the corresponding dominant sequence harmonic voltages (e.g. regarding the $5^{\text {th }}$ harmonic, the zero sequence voltage should be approx. the opposite of the negative sequence $5^{\text {th }}$ harmonic voltage measured on the busbar). In this case the harmonic current of the fault current will be near to zero.

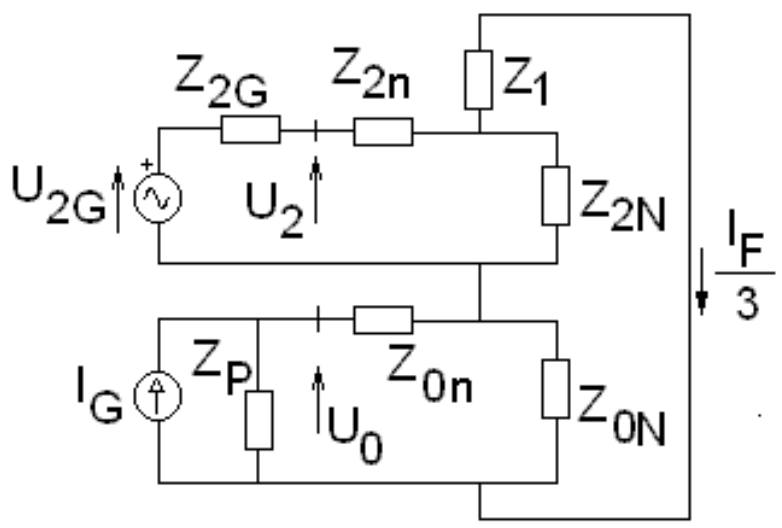

Fig. 8. Simplified positive-, negative and zero sequence network scheme for the $5^{\text {th }}$ harmonics in case of a single-line to ground fault

In Fig. $8 Z_{l}$ is the total positive sequence impedance, $Z_{2 G}$ is the negative sequence impedance of the supply system, $Z_{2 n}$ and $Z_{2 N}$ are the negative sequence impedances of the faulted line before and behind the fault location, $Z_{O n}$ and $Z_{O N}$ are the zero sequence impedances of the faulted line before and behind the fault location, $Z_{P}$ is the zero sequence impedance of the Petersen-coil, $U_{2}$ and 
$U_{0}$ are the negative and zero sequence voltages at the busbar, $I_{F}$ is the fault current. The dominant part of the $5^{\text {th }}$ harmonic currents and voltages in the non-faulted case composes a negative sequence system, therefore the $5^{\text {th }}$ harmonic voltage generator exists only in the negative sequence system. The zero sequence active filter can be modeled as a controlled current source $I_{G}$ connected in parallel to the Petersen coil.

The control algorithm seeks to minimize an error term $\Delta U$ in order to decrease the $5^{\text {th }}$ harmonic component in the fault current.

The following definition of $\Delta U$ will be used:

$$
\Delta \mathrm{U}=\left|-\underline{\mathrm{U}}_{0}-\underline{\mathrm{U}}_{2}\right|
$$

As an explanation of (1) consider, that

- $\quad Z_{l}$ can be neglected (i.e. the voltage drop at the positive sequence network is negligible)

- $Z_{0 n}$ can be neglected (as compared to $Z_{P}$ ), therefore $U_{0}$ approximately equals the voltage drop at $Z_{O N}$

Thus from Fig. 8 it can be seen, that if $-\underline{\mathrm{U}}_{0}=\underline{\mathrm{U}}_{2}$ then there will be no $5^{\text {th }}$ harmonic current flowing through the fault location.

Simulation and field test results are in good coincidence and will be presented in the final paper in greater detail.

The simulations shown below start with a negative sequence $5^{\text {th }}$ harmonic voltage of $U_{2 G}=150 \mathrm{Vrms}$. In order to simulate that the faulted line is not tripped and during operation the voltage of the supply network can change, $U_{2 G}=200 \mathrm{Vrms}$ (and a phase shift of $15^{\circ}$ ) is applied at the simulation step 50 instead of the original $150 \mathrm{Vrms}, 0^{\circ}$.

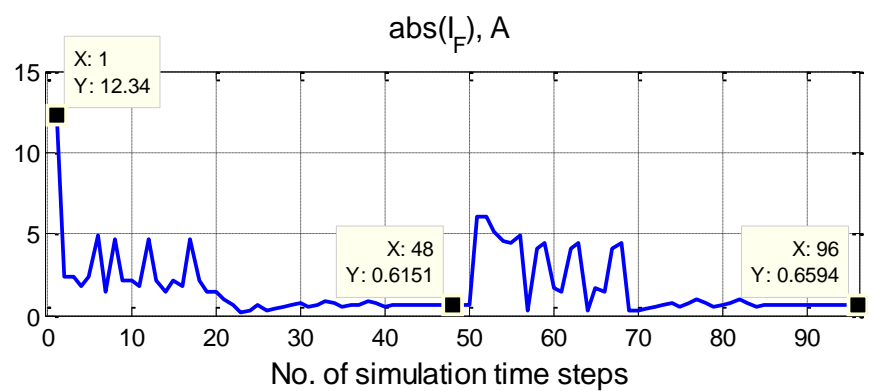

Fig. 9. Simulated $5^{\text {th }}$ harmonic content of the fault current

The on-site analysis showed that the $5^{\text {th }}$ harmonic content of the residual fault current was decreased (almost completely eliminated), and thus the RMS of the residual current was also decreased to around $3 \mathrm{~A}$.

\section{Conclusions}

Simulated and measured results show a good coincidence.

Equipment and methods presented are capable of

- fault location (with good accuracy) and of

- reducing the harmonic content of the residual current of single-phase to ground faults. (This allows the operators to maintain operation in case of single-phase to ground faults for several hours.)

Thus better continuity of supply indices (SAIDI and
SAIFI) can be achieved.

Test operation is underway in two substations.

\section{References}

[1] A.M. Dán, Zs. Czira: "Harmonics in Compensated Medium Voltage Overhead Systems during Single-phase to Earth Fault", Proceedings of the International Conference on Electric Power Quality and Supply Reliability, pp 122-125. 1999, Tallinn, Estonia

[2] H. Schau, D. Stade : Influence of harmonics on the line-toground fault currents. Ibid pp 116-121.

[3] András Dán, Zsuzsa Czira, Dávid Raisz: "Comparison of different methods to decrease the harmonic content of the fault current during single-phase to ground faults in compensated networks", IEEE PowerTech Conference 27-30 June, 2005, StPetersburg, Russia

[4] Janssen, M. Kraemer, S. Schmidt, R. Winter: "Residual current compensation (RCC) for resonant grounded transmission systems using high performance voltage source inverter", IEEE PES Transmission and Distribution Conference and Exposition, Sept. 7-12, 2003, pp. 574- 578

[5] Patent description number US 2007/0124093

[6] Patent description number EP 1304580

[7] Patent description number DE 10143595

[8] Patent description number EP 1089081

[9] Patent description number WO 97/08562

[10] Patent description number JP 4140016

[11] Patent description number DE 3016223

[12] Equipment and procedure to determine the fault location and the fault resistance during phase to ground faults on a live network with resonant or high impedance neutral, Patent pending, WO/2009/081215

http://www.wipo.int/pctdb/en/wo.jsp?WO=2009081215

\section{Biographies}

Dr. András M. Dán (SM'90) received MSc degree from Budapest Technical University in 1966, PhD and DSc degrees in Electrical Engineering from the Hungarian Academy of Sciences in 1983 and 2005 respectively. He has been at Budapest Technical University since 1970 where he currently holds the rank of professor and acts as a consultant for local industry. His expertise is in power electronics, power quality, reactive power compensation especially associated with power system harmonics. Dr Dán is a member of the Hungarian Electrotechnical Association

Dávid Raisz (M’06) was born in Budapest, Hungary in 1977. He graduated from the Department of Power Systems of the University of Technology and Economics Budapest, Hungary, in June 2000 after a one year research stay at Department for Electrical Power Systems of University of Technology Graz, Austria. His research interests are in power quality and AI applications.

$\mathrm{He}$ is now working towards his $\mathrm{Ph} . \mathrm{D}$. degree in Electrical Engineering as senior lecturer at the Budapest University of Technology and Economics. He is member of the Hungarian Electrotechnical Association.

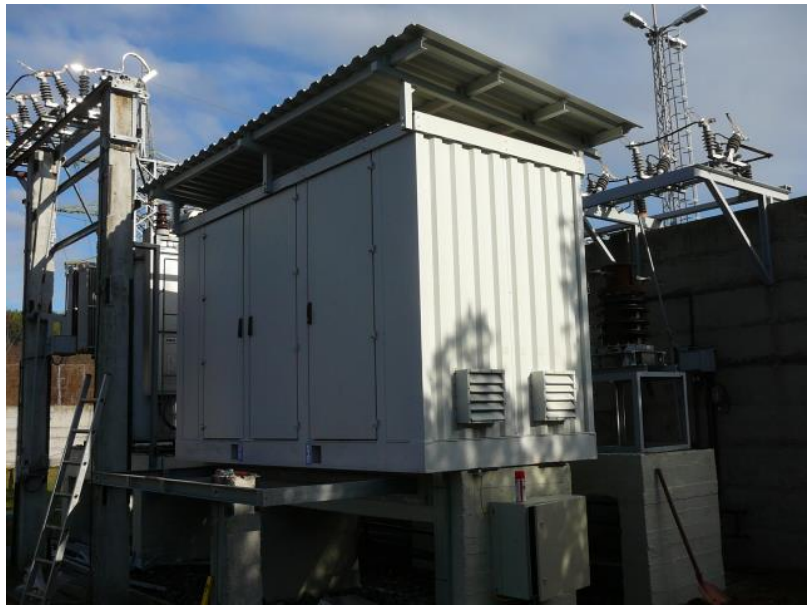

Fig. 10. Primary equipment at installation site No.2. 


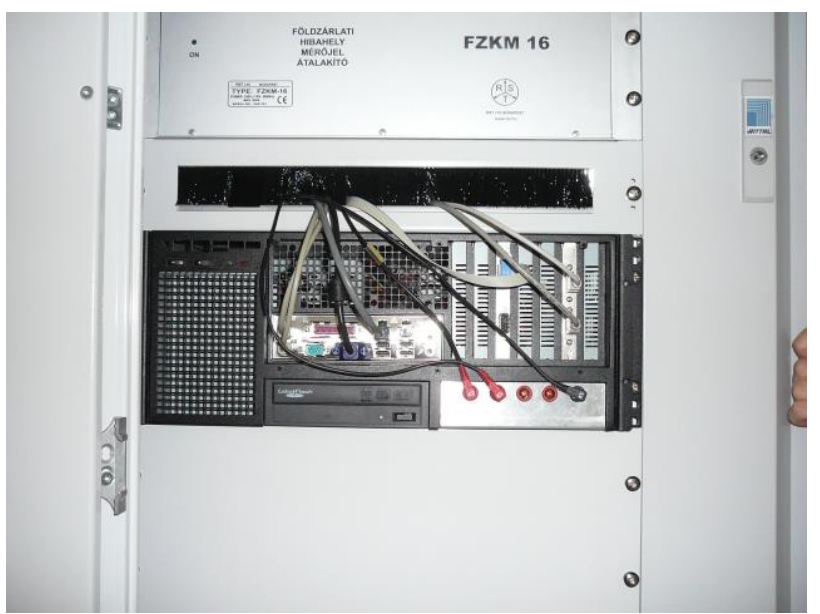

Fig. 11. Measuring equipment and industrial PC

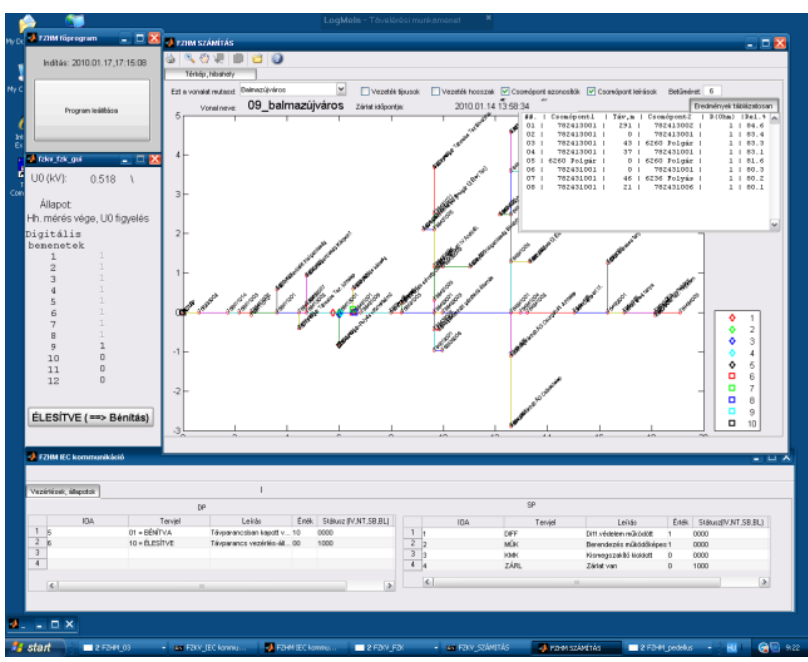

Fig. 12. Screenshot of the evaluation 